\title{
GPPS-BJ-2019-0069
}

\section{Pre-positioning of Optical Components for Pressure Sensitive Paint Measurement Using Prior Ray-tracing Method}

\author{
Tianyu Gao \\ Northwestern Polytechnical University \\ gaoty612@foxmail.com \\ Xi'an, Shaanxi, China
}

\author{
Limin Gao \\ Northwestern Polytechnical University \\ gaolm@nwpu.edu.cn \\ Xi'an, Shaanxi, China
}

\author{
Guanhua Yang \\ Northwestern Polytechnical \\ University \\ pk5434@mail.nwpu.edu.cn \\ Xi'an, Shaanxi, China
}

\author{
Tianlong Zheng \\ Northwestern Polytechnical \\ University \\ zhengtianlong@mail.nwpu.edu.cn \\ Xi'an, Shaanxi, China
}

\author{
Ning Ge \\ Northwestern Polytechnical \\ University \\ geningvip@163.com \\ Xi'an, Shaanxi, China
}

\section{ABSTRACT}

Measurement techniques like pressure sensitive paint (PSP) have shown their uniqueness in the capability of larger coverage of model surface area and higher spatial resolution in the results as compared with conventional ways. The mechanism of PSP technique requires the excitation light illuminates the entire test surface, and the camera records luminescent emission from the surface. Application of PSP technique may fail on internal flows like turbine and compressor cascades when these requirements are not met, where optical access may be heavily limited due to the closely adjacent components with curvature.

Such problems could only be overcome through trial and error, which could be time-consuming and requires accumulated experience. Therefore, a pre-positioning method is proposed in this work, which is based on ray-tracing simulations, to determine the arrangement of the optical components for PSP measurement system in advance. To verify the effectiveness of this method, it is used on a linear compressor cascade with large solidity. This method enables researchers to estimate the optical conditions and determine the optical devices' setup before the real test. The results show that with arrangement determined using this method proposed, the full-field pressure on a cascade blade surface with large solidity can be acquired for PSP technique. And results obtained have better uniformity of illumination with the same devices, which is beneficial for image quality and subsequent analyses of flow phenomena.

\section{INTRODUCTION}

Pressure measurement in wind tunnel testing is of great importance for the understanding of critical flow phenomena, such as shock and flow separation, which may offer comparison to computational fluid dynamics (CFD) predictions, and further facilitate the aerodynamic design. As a non-intrusive pressure measurement technique, pressure sensitive paint (PSP) has progressed rapidly over the past decades, and has matured to a point where many types of test models can be measured with a high spatial resolution in the results.

While PSP has been proved to be successful for the investigation of external flow like aircraft models in wind tunnel (Brown et al. 2000, Engler et al. 2001, Engler et al. 2002, Shimbo et al. 2000, Sellers et al. 2000), its applications to internal flow like turbomachines, however, are still complicated by various unresolved problems to date. One representative of them is the occlusion in the optical access through which to both illuminate and capture the test surfaces. Typically, a flow channel in a turbomachine is surrounded by four surfaces from two adjacent blades and upper and lower end-walls. Moreover, this situation is usually accompanied with complex geometries of the compressor or turbine blades. Thus, blockage of line of sight will be bothersome if the positions of PSP optical components are not carefully selected. As an image-based measurement technique, an appropriate setup of optical components is not only critical to the PSP image data collection, it also directly affects the subsequent data processing and the reliability of the pressure results.

Although the potential of PSP technique for investigating internal flow has been elucidated by many preceding studies, either on film cooling efficiency of turbine rotor or cascade (Ahn et al. 2006, Gao et al. 2008a, Gao et al. 2008b, Narzary et al. 2012, Chen et al. 2017, Li et al. 2016a, Li et al. 2016b, 
Peng et al. 2019), and pressure distribution studies on compressor blades (Lepicovsky et al. 2002, Zhou et al. 2009, Gao et al. 2011, Peng et al. 2017, Watanabe et al. 2018), the procedure of determining the position of optical components remains elusive. It can be cumbersome through trial and error seeking for an ideal arrangement for light sources to illuminate the test surfaces and for cameras to capture the PSP coated blade surfaces. What is even worse is that with higher solidity of cascade (where the narrowest part of the channel could be less than $2 \mathrm{~cm}$ ), it would be necessary to collect PSP images on one surface with multiple cameras (otherwise the pressure map acquired would be too skewed to extract data from within). Situations like that (Kurita et al. 2010) may require longer time to settle the devices' arrangement, with only empirical knowledge.

Therefore, a pre-positioning method based on Monte Carlo ray-tracing is developed in this work to help decide the spatial positions of the PSP optical devices. A linear compressor cascade with large solidity is used to verify the effectiveness of this pre-positioning method. The results show that this method enables researchers to understand the optical environment better in advance, and the illuminance distribution on the test surface can be obtained through simulation beforehand to benefit the PSP measurement.

\section{PROPOSED PRE-POSITIONING METHOD}

Monte Carlo ray-tracing method has been widely used to calculate the performance of optical systems. In this current paper, we conduct numerical ray-tracing simulations with some adaptations in order to analyze the optical components arrangement for the PSP measurement system. Seeking for positions of the optical components is like the inverse problem of directly simulating the trace of each ray. The spatial location and tilt of each light source and camera, as the final objectives of this current work, are unknown to the researchers so that one cannot perform the ray-tracing as it usually does in an optical system design. Therefore, in this proposed method, some requirements are given so that ray-tracing could fit in with the selection of positions for optical components, and the results could be then evaluated both qualitatively and quantitatively.

This pre-positioning method consists of four major steps, i.e. modeling the test optical environment, defining reference surface, ray-tracing and analyzing the illumination.

\subsection{Definition of the optical component setup for PSP measurement system}

Based on the working principle of PSP technique (Liu et al. 2006), a PSP measurement system generally includes test surfaces coated with pressure sensitive paint coating, optical components include light source of proper wavelength to excite the coating, and detector like CCD camera to collect the luminescent emission, as shown in Fig. 1. Prior to a PSP experiment, the spatial locations of the light source and camera have to be settled reasonably to ensure the coated surfaces covered with excitation light and can be collected by cameras.

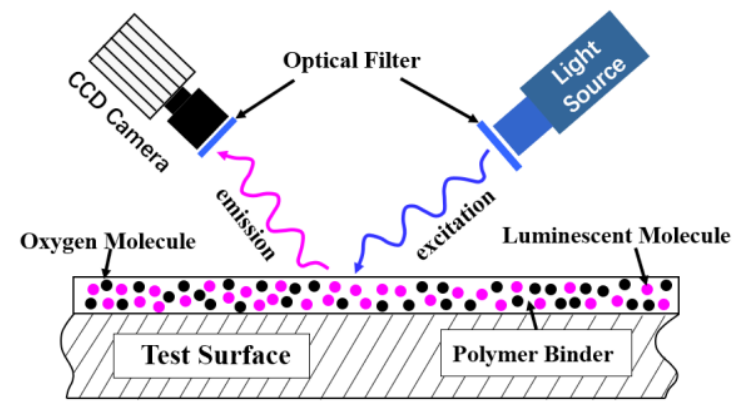

Figure 1 Schematic of PSP measurement system

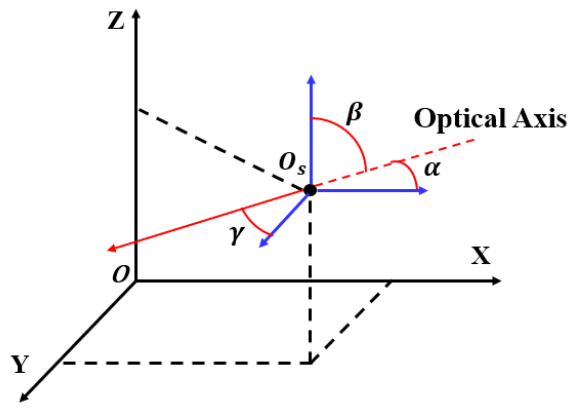

Figure 2 Spatial location of light source/camera

The spatial locations $(x, y, z)$ and azimuth angles $(\alpha, \beta$, $\gamma$ ) of the camera and light source are all described as shown in Fig. 2 in Cartesian coordinates originated from the geometric centre $O_{S}$ of the test surface, and azimuth angles can be determined by the corresponding spatial locations, as described in Eq. (1):

$$
\left\{\begin{array}{l}
\alpha=\arccos \left(\frac{\left|x-x_{O_{S}}\right|}{\sqrt{\left(x-x_{O_{S}}\right)^{2}+\left(y-y_{O_{S}}\right)^{2}+\left(z-z_{\left.O_{S}\right)^{2}}\right.}}\right) \\
\beta=\arccos \left(\frac{\left|y-y_{O_{S}}\right|}{\sqrt{\left(x-x_{O_{S}}\right)^{2}+\left(y-y_{O_{S}}\right)^{2}+\left(z-z_{\left.O_{S}\right)^{2}}\right.}}\right) \\
\gamma=\arccos \left(\frac{\left|z-z_{O_{S}}\right|}{\sqrt{\left(x-x_{O_{S}}\right)^{2}+\left(y-y_{O_{S}}\right)^{2}+\left(z-z_{O_{S}}\right)^{2}}}\right)
\end{array}\right.
$$

\subsection{Optical requirements for the PSP Measurement System}

To obtain a qualified PSP image, some requirements for the excitation light from light sources and emission light collected by cameras should to be discussed in detail.

Based on the quenching mechanism of luminescent paintings, for getting luminescent molecules immersed in PSP coatings on the test surface excited, the illuminance $E$ from the light source should reach every corner of the test surface $S_{A B C D}$ as presented in Fig. 3, and all should exceed the minimum required illuminance $\boldsymbol{E}_{\text {limit }}$. Thus, the first requirement for light source can be expressed by Eq. (2):

$$
\left\{E_{A}, E_{B}, E_{c}, E_{D}\right\}_{\text {min }} \geq E_{\text {limit }}
$$




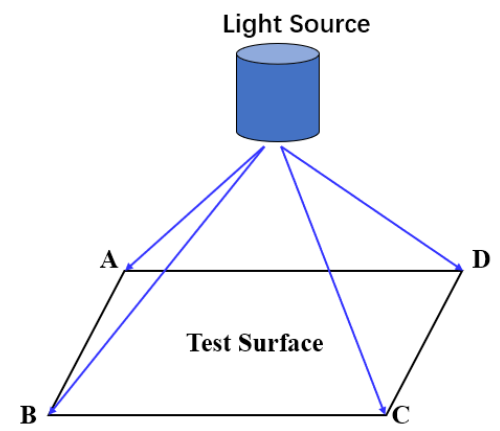

Figure 3 Requirement of illuminance for light source

To lower the influence of illumination nonuniformity on the test surface, the illumination uniformity $\boldsymbol{U}_{\boldsymbol{E}}$ should be confined above a certain level as described by Eq. (3):

$$
U_{E} \geq U_{\text {Elimit }}
$$

And Eq. (4):

$$
U_{E}=E_{\min } / E_{\max }
$$

Here the illumination uniformity $\boldsymbol{U}_{\boldsymbol{E}}$ is defined as the ratio of the maximum illuminance $\boldsymbol{E}_{\boldsymbol{m a x}}$ and minimum illuminance $\boldsymbol{E}_{\text {min }}$ across the entire test surface.

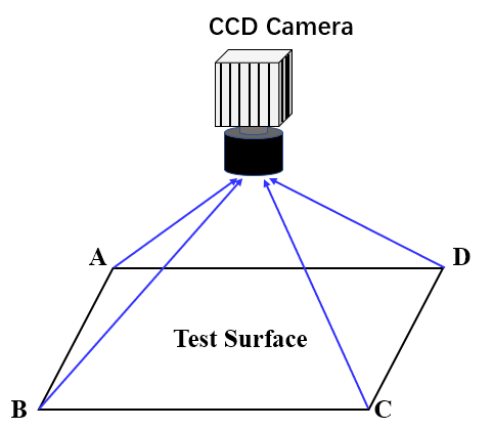

Figure 4 Requirement of coverage area for camera

As for the recording camera shown in Fig. 4, it is required that all fluorescence $\boldsymbol{I}$ emitted from the paintings on test surface be detected by the camera. In a practical PSP measurement, emission light from paintings may be contaminated by the surrounding environment. Therefore, to have a good SNR (signal to noise ratio) in images, the emission intensity from coatings on the test surface $S_{A B C D}$ should also be assured to exceed the noise intensity $\boldsymbol{I}_{\text {limit }}$, this is described in Eq. (5):

$$
\left\{I_{A}, I_{B}, I_{C}, I_{D}\right\}_{\text {min }} \geq I_{\text {limit }}(5)
$$

The visual angle of a camera directly affects the model deformation in photos. An ideal in photography is that the optical axis of a camera lens is perpendicular to the test surface. However, in a practical PSP experiment, especially for compressor cascade, it is too difficult to meet this perpendicular condition due to structural limits. It is very common in internal flow measurements that test surfaces are blocked partially and even entirely in the photos. Therefore, viewing angle $\theta$ in Eq. (6) between the test surface's normal vector $\vec{n}$ and the camera lens axis $\vec{l}$ should be minimized as described in Eq. (7):

$$
\begin{gathered}
\theta=\arccos \left(\frac{\vec{l} \cdot \vec{n}}{|\vec{\imath}| \cdot|\vec{n}|}\right) \\
\left\{\theta_{A}, \theta_{B}, \theta_{C}, \theta_{D}\right\}_{\text {max }}<\theta_{\text {limit }}
\end{gathered}
$$

\subsection{Numerical Ray-tracing Methodology}

The non-sequential ray tracing based on Monte Carlo method is used to resolve the illumination simulation, basic procedures of which are demonstrated in Fig. 5.

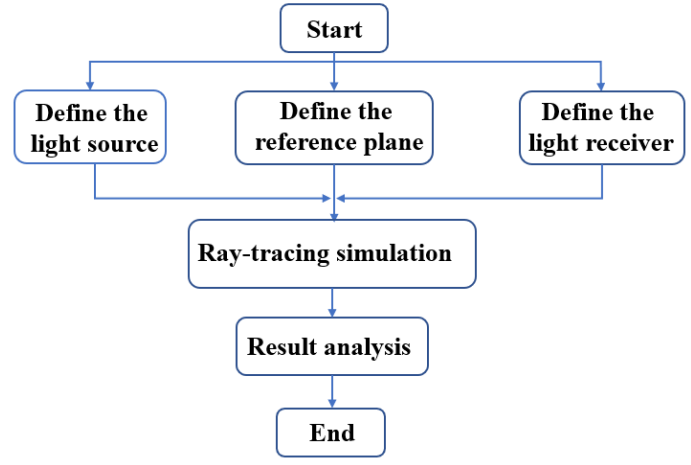

Figure 5 Basic procedures of ray-tracing simulation

Our proposed method is similar to the design of optical systems using ray tracing, as described in the preceding sections, it mainly consists of four steps, 3D modeling, light source and light receiver definition, ray tracing simulation and the final result analysis.

Firstly, the wind tunnel test rig and optical devices need to be $3 \mathrm{D}$ modeled digitally for ray-tracing simulations. While building up the models the coordinate system of each one should be kept consistent. Secondly, a light source in ray tracing simulation can be defined by selecting from a point source, a line source, or a surface source (including a planar light source, a curved light source, or an array light source, etc.) according to the needs of researchers. Thirdly, a light receiver is generally a plane or a curved surface in space, with many parameters to be selected such as light transmittance, reflectivity and absorptivity. These parameters can be selected according to the optical characteristics of the actual objects. And after completing the above steps, ray tracing simulation and result analysis can be finally performed.

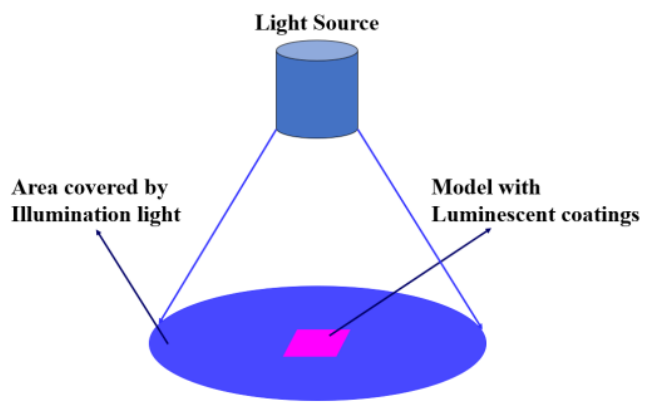

Figure 6 A test surface receives simulated rays

It is important to note, however, that the total number of simulated rays in the ray-tracing program is anyhow limited compared with in reality. And since a tested cascade blade 
surface has a much smaller area compared with the coverage of illumination light, it may receive only small part of the simulated rays, this situation can be described as shown in Fig. 6. And if so the uniformity of illumination calculated would be inappropriate. Therefore, the total number of simulated rays should be sufficient to avoid bias. And since the calculation time will increase as the number of light rays increases, it would be appropriate to set the total number of rays to around 25,000 , based on extensive testing.

Additionally, two more factors need to be considered during pre-positioning:

1) In a practical experiment, the installation of the CCD camera will take up a certain volume of space, thus a distance limit should be kept from the camera to the measurement model to avoid interference.

2)When determining the spatial position of the CCD camera and light source, these two devices should not interfere with each other.

\subsection{Procedures of the Proposed Pre-positioning Method}

The procedures of the proposed pre-positioning method for determining the spatial location of the camera and light source is shown in Fig. 7.

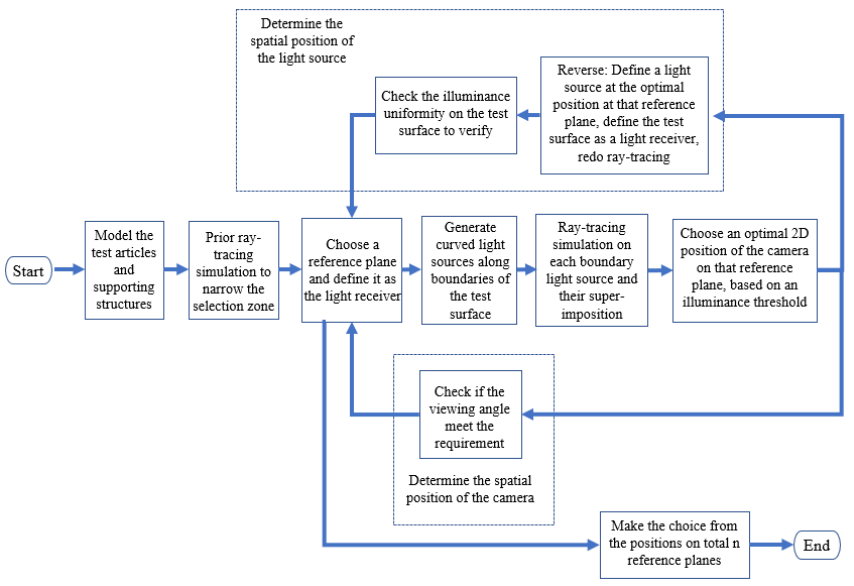

\section{Figure 7 Procedures of the proposed pre- positioning method}

Digitally modeling the test rig and optical devices would still be the very first step of the whole process. Since there may exist interference between the camera and light source in the limited available space, spatial coordinates of the camera $\left(x_{C}, y_{C}, z_{C}\right)$ are firstly determined in this method. And because the distance from the camera's aperture to the chip is quite small compared with the distance to the test surface, the spatial coordinates of the camera will be approximated by its aperture's.

A prior simulation to the $3 \mathrm{D}$ models would help narrow down the range of choices for positions. Now in the narrowed range we need more ray-tracing to seek for specific locations $\left(x_{C}, y_{C}, z_{C}\right)$ for the camera and $\left(x_{L}, y_{L}, z_{L}\right)$ for the light source (where subscript $C$ denotes camera and subscript $L$ denotes light source).

Before the extensive ray-tracing, some simplifications are introduced to facilitate pre-positioning. The determination of
3D positions will be simplified into $2 \mathrm{D}$ positions and then back to 3D: one of the three unknown coordinates of the camera/light source need to be firstly fixed (e.g. $x_{C}$ is fixed as $x_{C 0}$ ), thus the problem will be simplified to determine the two-dimensional coordinates $\left(y_{C}, z_{C}\right)$ in an already known plane $x=x_{C 0}$ (we call this plane as the reference plane), and this reference plane will be defined as light receiver in the raytracing program to collect illumination. In this way, the optimal location of the camera/light source is assumed to be in this reference plane $x=x_{c 0}$. After that, add another reference plane that is parallel to the former one with a different distance to the tested model, and redo the ray-tracing and location finding again, the 3D spatial positions of camera/light source can be settled down progressively.

For the subsequent ray-tracing part of this pre-positioning method, simply define the entire test surface as illuminant to inversely find the positions will certainly do, but that will cost extra computing resource. In fact, consider this, if emission light from all boundaries of the test surface can be collected by the camera, and if rays from the light source can reach every boundary of the surface, it is safe to assume that the arrangement works. Therefore, the concept of boundary light source is introduced here, as shown in Fig. 8, which plays the role of representative of the entire surface. Boundaries will be defined as illuminant to do the ray-tracing, and their light divergence mode will be selected as Lambertian.
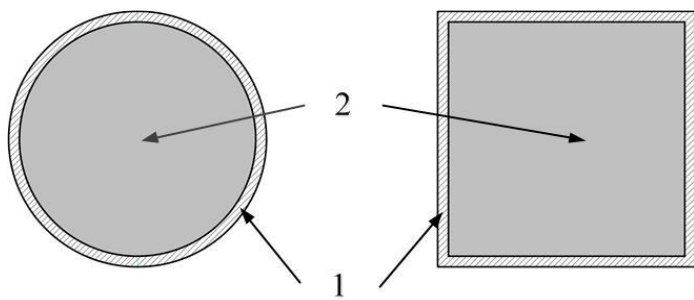

Figure 8 Schematic of boundary light sources (number 1 represents the boundaries, and number 2 represents the major part of surfaces)

Ray-tracing simulation is performed each time with every single boundary light source defined to obtain the illuminance distribution on the reference plane. Adding up the results from each boundary light source and normalize it with its peak value, this will give a dimensionless illuminance distribution on the reference plane. With a threshold selected according to the requirement of $\boldsymbol{E}_{\text {limit }}$, large area on one reference plane will be narrowed down to a smaller region, and in this region the location with maximum illuminance $\left(x_{c m}, y_{c m}, z_{c m}\right)$ would become an alternative choice for putting camera. However, it has to be checked whether the viewing angle $\theta$ here can meet the requirement described in section 2.2. If not, then the position has to be altered with as higher illuminance as possible in the narrowed region.

One additional step for determining the light source is to put the light source here at the location picked, and redefine the test surface as the light receiver. The purpose of this is to check whether the illumination uniformity $\boldsymbol{U}_{\boldsymbol{E}}$ is okay. If so, this location calculated $\left(x_{l m}, y_{l m}, z_{l m}\right)$ will be good to put the camera and light source, though need some tweak to accommodate both two devices, while camera has the priority. 
In this way, if reference planes $x=x_{1}, x_{2}, \ldots, x_{n}$ are defined, we can get totally $n$ sets of positions for setting up optical devices. An optimal position could be settled from these optional positions by evaluating the illuminance distribution on the reference plane and the illumination uniformity on the test surface.

\section{RESULTS AND DISCUSSION}

\subsection{Test Rig and Compressor Cascade}

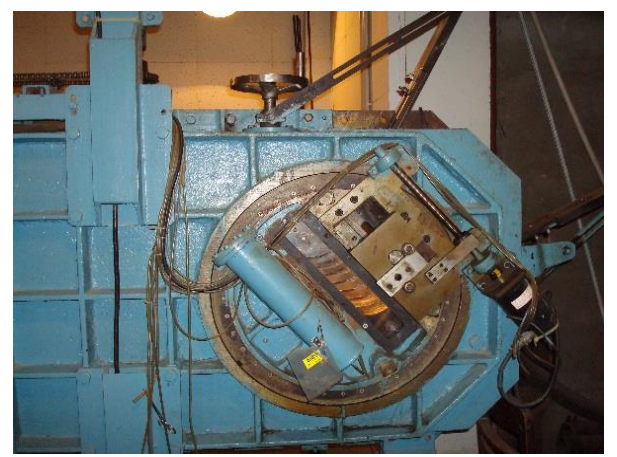

Figure 9 Test section of high-subsonic cascade tunnel

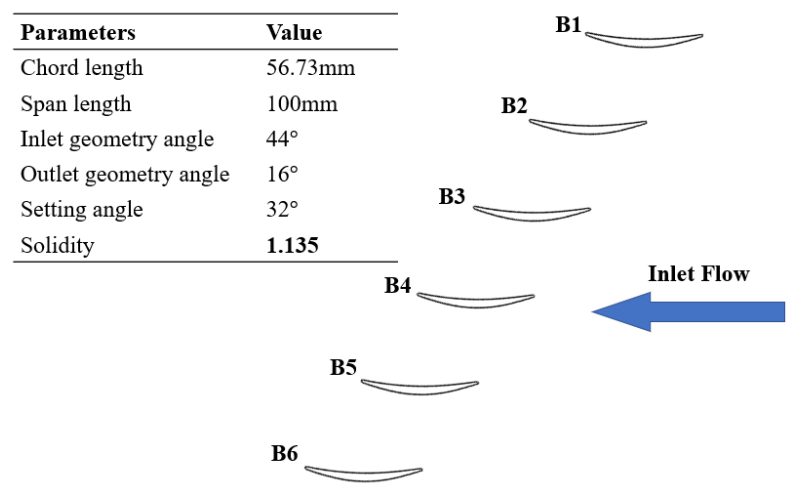

Figure 10 Test compressor cascade

To verify the effectiveness of the proposed method, we perform the pre-positioning to the identical test rig and optical devices used in our previous work (Gao et al. 2011), in that work the PSP technique was used to acquire the pressure distribution on the suction surface of a linear compressor cascade.

This high-subsonic linear cascade wind tunnel used is as shown in Fig. 9. The test section is rectangular and has a $100 \times 300 \mathrm{~mm}$ cross section. All the six blades in the compressor cascade has a span of $100 \mathrm{~mm}$ and a chord length of $56.73 \mathrm{~mm}$. Detailed parameters and a schematic view of the test cascade are given in Fig. 10. This research finally obtained the pressure distribution on the suction side surface of a cascade blade under the inlet Mach number of $M a=0.4$ and $M a=0.5$.

\subsection{Verification of the Pre-positioning Method}

According to the procedures described in preceding sections, the three-dimensional solid models of all components of the test rig are firstly built, including simplified model of the wind tunnel test section and the linear cascade. Then the models are imported into the ray-tracing program and their surface optical properties are selected reasonably.

Before accurately determine the spatial location of the light source and camera, a larger coarse area need to be firstly selected. Therefore, the ray-tracing simulation is firstly carried out by setting the test suction surface of the blade as the surface light source, and the light ray distribution shown in Fig. 11 is obtained. As can be seen, a large number of rays are concentrated in both sides and the exit of the linear cascade wind tunnel, this is because the end wall on both sides are replaced by the acrylic sheets for optical access, and no obstruction is at the exit of the wind tunnel. There are three alternative regions for the arrangement of the light source and the camera. And since the optional regions B and C on both sides of the wind tunnel are approximately symmetrical, only figuring out the spatial positions for the optical components in the region $\mathrm{A}$ and $\mathrm{C}$ would be necessary.

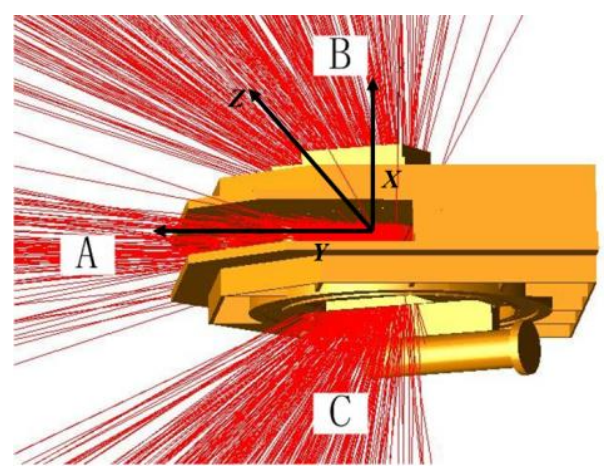

\section{Figure 11 A prior ray-tracing simulation to narrow the selection zone for optical components}

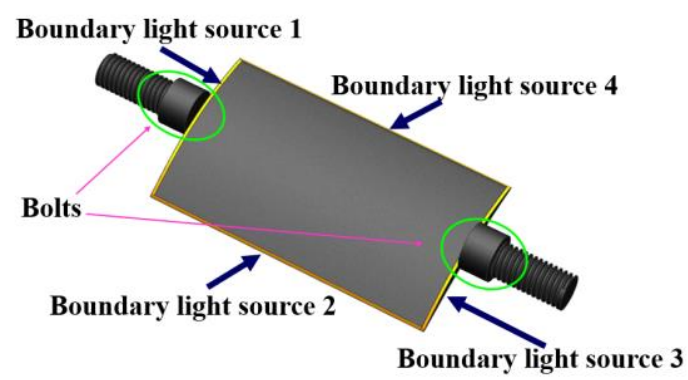

\section{Figure 12 Boundary light sources of a cascade blade}

The coordinate system of this simulation is defined as follows, the geometric center of the suction surface of the test blade is chosen as the origin of the coordinate system, as shown in Fig. 11, wherein the $\mathrm{Z}$ axis is perpendicular to the ground pointing upwards and the $\mathrm{X}$ axis is perpendicular to the sidewall of the wind tunnel, and $Y$ axis pointing to the exit of the cascade tunnel.

Since the distance from the CCD camera to the measured cascade vanes is limited by the size of the camera itself, the reference plane $x=-400 \mathrm{~mm}$ is selected as the closest one from the surface of the test blade, and a large flat plate is built on this reference plane as a light receiver. The surface boundary curved light source of the measured cascade vane is defined as shown in Fig. 12. A total number of around 30,000 
rays is set originated from the light sources. Illuminance distribution on the reference plane under different boundary curved light source is then obtained through ray-tracing simulation, and the corresponding spatial coordinates at the maximum illuminance is estimated to be $(-400 \mathrm{~mm}$, $156.25 \mathrm{~mm},-156.25 \mathrm{~mm}$ ), azimuths are estimated to be $\left(32.57^{\circ}, 69.69^{\circ}, 114.3^{\circ}\right)$, while the angle $\theta$ between the normal vector $\vec{n}$ at the geometric center of the surface of the cascade vane and the corresponding vector $\vec{l}$ of the camera lens optical axis is estimated to be $59.82^{\circ}$. After that, four more reference planes parallel to the first one $x=-400 \mathrm{~mm}$ are selected and the $3 \mathrm{D}$ coordinates and azimuths are calculated. The results of the five groups are finally shown in Table. 1.

\begin{tabular}{|c|c|c|c|c|c|}
\hline$x / m m$ & -400 & -600 & -800 & -1000 & -1200 \\
\hline$y / m m$ & 156.25 & 234.37 & 312.50 & 390.63 & 429.69 \\
\hline$z / m m$ & -156.25 & -296.88 & -312.50 & -390.63 & -468.75 \\
\hline $\boldsymbol{\alpha} /^{\circ}$ & 32.57 & 31.36 & 30.76 & 30.39 & 29.20 \\
\hline $\boldsymbol{\beta} /^{\circ}$ & 69.69 & 69.78 & 69.83 & 69.86 & 71.41 \\
\hline $\boldsymbol{\gamma} /^{\circ}$ & 65.70 & 67.10 & 67.81 & 68.24 & 68.32 \\
\hline $\boldsymbol{\theta} /^{\circ}$ & 59.82 & 61.24 & 61.96 & 62.40 & 63.02 \\
\hline
\end{tabular}

Table 1 Spatial coordinates and angles of camera

Similarly, ray-tracing simulation is also carried out to give five alternative positions putting light sources. Compared with camera, illuminance uniformity on the test surface is further calculated according to $E q$. (4), and the results are given in Table. 2.

\begin{tabular}{|c|c|c|c|c|c|}
\hline$x / \mathrm{mm}$ & 0 & 0 & 0 & 0 & 0 \\
\hline$y / \mathrm{mm}$ & 500 & 600 & 700 & 800 & 900 \\
\hline$z / \mathrm{mm}$ & -62.50 & -93.75 & -125 & -125 & -125 \\
\hline$\alpha \rho$ & 90 & 90 & 90 & 90 & 90 \\
\hline$\beta \rho$ & 11.68 & 12.66 & 13.35 & 11.72 & 10.43 \\
\hline$\gamma \rho$ & 78.32 & 77.34 & 76.65 & 78.28 & 79.57 \\
\hline$U_{E}$ & 0.2044 & 0.0988 & 0.1166 & 0.1689 & 0.2417 \\
\hline
\end{tabular}

Table 2 Spatial coordinates and angles of light source

It should be noted that, according to preceding sections, coordinate $y=900 \mathrm{~mm}$ that corresponds to the maximum value of illuminance uniformity $U_{E}$ should be selected intuitively as the optimal position for the light source. However, this light source used has a somehow scattered ray distribution, thus it may cause insufficient illumination on the test surface once the distance between the light source and cascade vanes becomes large. So instead coordinate of $y=$ $500 \mathrm{~mm}$ is finally selected.

Based on the above simulation results, the arrangement for optical components is digitally modeled for observation, as presented in Fig. 13. While in comparison, the arrangement of the components on the scene is given in Fig. 14.

It can be seen that the spatial position and tilt of camera are basically consistent between the simulation and the actual arrangement, though slight difference exist in the position of the light source. Previously in our study, this light source has a smaller coordinate in $\mathrm{Z}$ axis. Although this would benefit the illuminance value around leading edge of the cascade vane, illuminance at the trailing edge of the blade, however, would then be reduced, so as its uniformity across the entire surface from the leading edge to the trailing edge. As discussed before, a better illuminance uniformity needs to be ensured throughout the pre-positioning process. Therefore, the optical components arrangement based on the proposed method in the current work is believed to be reasonable in a sense.

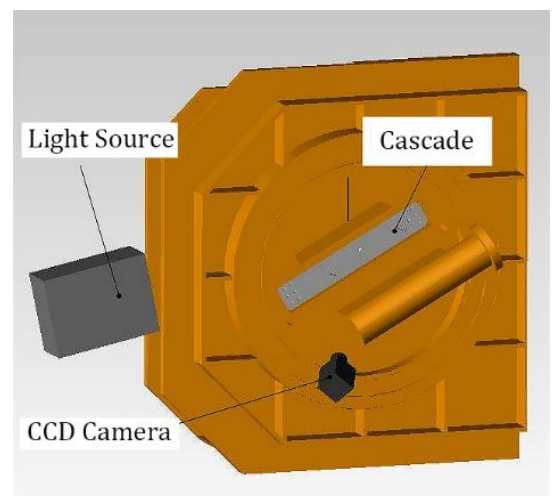

\section{Figure 13 Optical components arrangement determined by ray-tracing simulation}

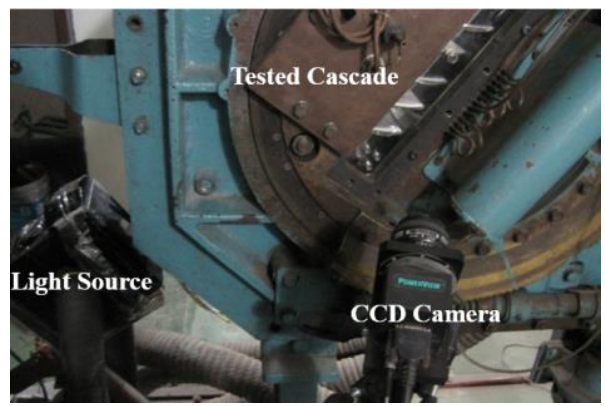

\section{Figure 14 Optical components arrangement in actual measurement test}

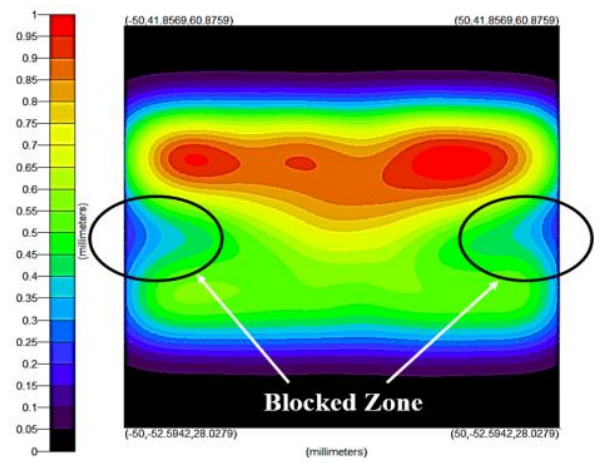

Figure 15 Simulated illuminance on the blade

Additionally, to check the effectiveness of the prepositioning method proposed, a light source putting at where the results point to is defined in the ray-tracing program, and the calculated distribution of illuminance on the cascade vane surface is shown in Fig. 15. For simplicity, illuminance across this surface is normalized by its maximum value. As can be seen, light from the source is able to illuminate substantially the entire suction surface of the vane, whereas two symmetrical areas at around the middle chord of the vane are spotted with illuminance heavily decreased. This is caused by the protruding bolts attached to the vane (see Fig. 12). Experiment results also coincide on this phenomenon as shown in Fig. 16. Unfortunately, this effect can never be eliminated so long as the bolts exist. The pressure-converted 
result is given in Fig. 17, while the comparison between the PSP results and pressure taps along the midspan of the blade is presented in Fig. 18, this two agree well with each other.

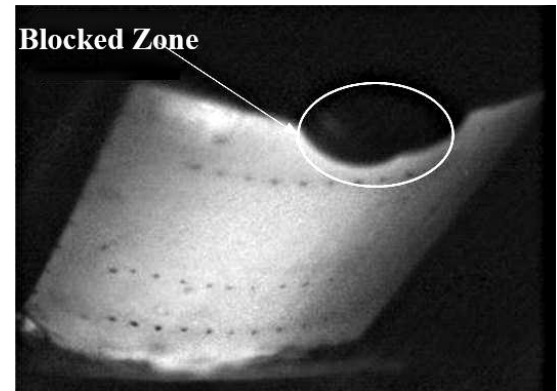

Figure 16 Raw PSP image of the blade

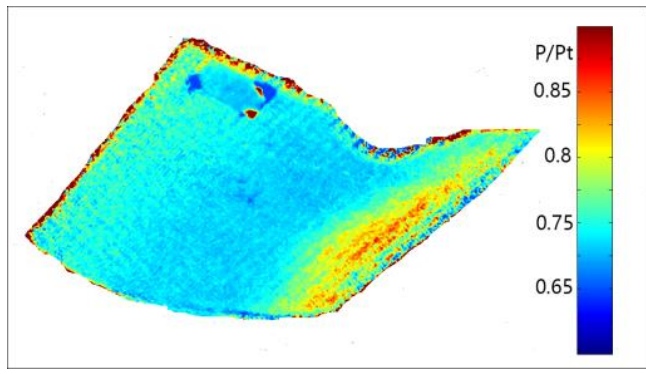

Figure 17 Pressure-converted image of the vane

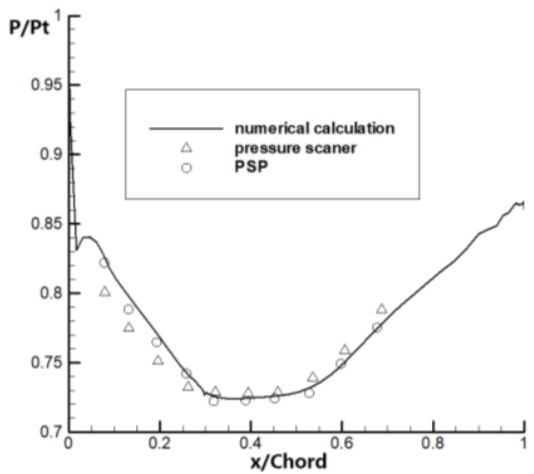

\section{Figure 18 Comparison between PSP and pressure taps}

To sum up, the pre-positioning method proposed in this current paper enables researchers to understand the experimental environment for PSP test with more information in advance, thus it can reduce the time spent in the trial and error process where no previous knowledge is involved. In the meantime, the illuminance distribution on the test surfaces can be obtained beforehand to benefit the PSP measurement.

Nevertheless, we have to admit that the process of $3 \mathrm{D}$ modeling and ray-tracing simulation may add extra time cost to the whole PSP experiment. Moreover, it could be inconvenient to determine the relative spatial positions of the optical devices to the test model. Still, there is room for improvement to this pre-positioning method in the way of matching up with practical applications. One possible solution is to expand a certain spatial position to a small confined region in space. Effort is being made to make this method more flexible and wieldier.

\section{CONCLUSIONS}

In this current paper, a pre-positioning method based on ray-tracing simulation is proposed to quantitatively determine the optical components setup for the PSP measurement before the real test. It is then used to determine the optical components arrangement of a linear compressor cascade tunnel. Comparisons are made between the simulation results and the real test. The conclusions are as follows:

(1) This proposed pre-positioning method can well determine the optical components arrangement in advance both qualitatively and quantitatively.

(2) More options of optical components arrangement can be provided based on this pre-positioning method; thus, the efficiency of PSP measurement experiment can be improved significantly.

(3) Blocked zones of illumination light on the test surface can be spotted in advance using this prepositioning method, which can lower the risk brought by uneven illumination.

\section{NOMENCLATURE}

$E \quad$ Illuminance on the test surfaces

$E_{\text {limit }} \quad$ Required minimum illuminance on the test surface to excite PSP coatings

$U_{E} \quad$ Illumination uniformity on the test surface

$E_{\max } \quad$ Maximum illuminance on the test surface

$E_{\min } \quad$ Minimum illuminance on the test surface

I Fluorescence emitted from PSP coatings

$I_{\text {limit }} \quad$ Intensity of noise in the fluorescence

SNR Signal to noise ratio

\section{ACKNOWLEDGMENTS}

The authors express their sincere gratitude to Mrs. Chen Liusheng, a researcher in the Institute of Chemistry at the Chinese Academy of Sciences, for providing the pressure sensitive paint used in our previous study. This study was cosupported by the National Natural Science Foundation of China (No. 51790512), the Overseas Expertise Introduction Project for Discipline Innovation (No. B17037) and the financial support of MIIT.

\section{References}

Ahn, J., Schobeiri, M. T., Han, J. C., \& Moon, H. K. (2006). Film cooling effectiveness on the leading edge region of a rotating turbine blade with two rows of film cooling holes using pressure sensitive paint. Journal of heat transfer, 128(9), 879-888.

Bencic, T. (1998, January). Rotating pressure and temperature measurements on scale-model fans using luminescent paints. In 34th AIAA/ASME/SAE/ASEE Joint Propulsion Conference and Exhibit (p. 3452).

Brown, O. C. (2000). Low-speed pressure measurements using a luminescent coating system.

Chen, A. F., Shiau, C. C., \& Han, J. C. (2017). Turbine blade platform film cooling with simulated swirl purge flow and slash face leakage conditions. Journal of Turbomachinery, 139(3), 031012. 
Engler, R. H., Klein, C., Merlo, E., \& van Amerom, P. (2001, August). 360/spl deg/PSP measurements in transonic flow. In ICIASF 2001 Record, 19th International Congress on Instrumentation in Aerospace Simulation Facilities (Cat. No. 01CH37215) (pp. 149-158). IEEE.

Engler, R. H., Mérienne, M. C., Klein, C., \& Le Sant, Y. (2002). Application of PSP in low speed flows. Aerospace Science and Technology, 6(5), 313-322.

GAO, L. M., GAO, J., WANG, H., ZHOU, Q., \& LIU, B. (2011). PSP experiment of global surface pressure distribution on suction surface of compressor cascade with large curved angle. Journal of Aerospace Power, 9.

Gao, Z., Narzary, D. P., \& Han, J. C. (2008). Film cooling on a gas turbine blade pressure side or suction side with axial shaped holes. International Journal of Heat and Mass Transfer, 51(9-10), 2139-2152.

Gao, Z., Narzary, D., Mhetras, S., \& Han, J. C. (2008). Full-coverage film cooling for a turbine blade with axialshaped holes. Journal of Thermophysics and Heat Transfer, 22(1), 50-61.

Kurita, M., Mitsuo, K., Nakakita, K., \& Watanabe, S. (2010). Multi-Camera Pressure-Sensitive Paint Measurement. In 27th AIAA Aerodynamic Measurement Technology and Ground Testing Conference (p. 4797).

Lepicovsky, J., \& Bencic, T. (2002). Use of pressuresensitive paint for diagnostics in turbomachinery flows with shocks. Experiments in Fluids, 33(4), 531-538.

Li, X., Ren, J., \& Jiang, H. (2016). Multi-row film cooling characteristics on a vane endwall. International Journal of Heat and Mass Transfer, 92, 23-33.

Li, X., Ren, J., \& Jiang, H. (2016). Influence of different film cooling arrangements on endwall cooling. International Journal of Heat and Mass Transfer, 102, 348-359.

Liu, T., \& Sullivan, J. P. (2006). Pressure and Temperature Sensitive Paints. Springer Science \& Business Media.

Narzary, D. P., Liu, K. C., Rallabandi, A. P., \& Han, J. C. (2012). Influence of coolant density on turbine blade filmcooling using pressure sensitive paint technique. Journal of Turbomachinery, 134(3), 031006.

Peng, D., Jiao, L., Yu, Y., Liu, Y., Oshio, T., Kawakubo, T., \& Yakushiji, A. (2017). Single-shot lifetime-based PSP and TSP measurements on turbocharger compressor blades. Experiments in Fluids, 58(9), 127.

Peng, D., Zhong, Z., Gu, F., Zhou, W., Qi, F., \& Liu, Y. (2019). Pressure-sensitive paint with imprinted pattern for full-field endoscopic measurement using a color camera. Sensors and Actuators A: Physical, 290, 28-35.

Shimbo, Y., Asai, K., \& Komatsu, N. (2000, August). Pressure sensitive paint application at large production wind tunnels. In proceedings of 22nd congress of international council of the aeronautical sciences, Paper No. 00-3.3.

Sellers, M. E. (2000, June). Application of pressure sensitive paint for determining aerodynamic loads on a scale model of the F-16C. In 21st Aerodynamic Measurement Technology and Ground Testing Conference (p. 2528).

Watanabe, T., Azuma, T., Uzawa, S., Himeno, T., \& Inoue, C. (2018). Unsteady pressure measurement on oscillating blade in transonic flow using fast-response pressure-sensitive paint. Journal of Turbomachinery, 140(6), 061003.

Zhou, Q., Liu, B., Gao, L., Chen, L., \& Shi, M. (2009). Pressure measurement on suction surface of a single vane using pressure-sensitive paint. Chinese Journal of Aeronautics, 22(2), 138-144. 